\title{
Eye blink reflexes to sudden free falls: a clinical test of otolith function
}

\author{
G MICHAEL HALMAGYI, MICHAEL A GRESTY \\ From the Medical Research Council, Neuro-Otology Unit, Institute of Neurology, National Hospital for \\ Nervous Diseases, Queen Square, London, UK
}

SUMMARY Conventional neuro-otological tests measure only semi-circular canal function and not otolith function. A clinically acceptable test of otolith function was developed. Previous studies suggested that muscle responses which occur less than $100 \mathrm{~ms}$ after release into free-fall are part of a startle reflex originating in the otoliths. With a couch capable of producing sudden, safe, comfortable free-falls it was shown that in normal subjects orbicularis oculi muscle responses (that is eyeblinks) invariably began within $45 \mathrm{~ms}$ of this stimulus and confirmed that these eyeblink reflexes are part of a generalised startle reflex, as they cross-habituate with eyeblink reflexes elicited by supraorbital nerve stimulation. Eyeblink reflexes to free-fall may prove a useful clinical test of otolith function.

The human vestibular system comprises three pairs of semi-circular canals and two pairs of otolith organs-the utricle and the saccule. Despite past efforts to develop clinical tests for each vestibular organ, only tests of horizontal semi-circular canal function have so far gained general acceptance. The otoliths respond to linear acceleration and it therefore seemed obvious to try and use changes in gravitational force to stimulate the otoliths. Previous reports suggested that early muscle response to sudden free-fall originate in the otoliths. ${ }^{1-3}$ We have therefore tried to reproduce these results in patients and normal subjects in a way that could be useful in neuro-otological diagnosis.

\section{Materials and methods}

\section{Apparatus}

Each subject and patient lay on a $10 \mathrm{~cm}$ thick, foam rubber mattress, inside a specially constructed wooden couch (fig 1). The foot of the couch pivoted about an axle, joining a pair of bicycle wheels; the head of the couch could be elevated by a winch and pulley arrangement and secured in an elevated position with a nautical snatch cleat. Upon releasing the cleat, the head of the couch (and the head of the patient) dropped freely through a vertical height of 12 cm (angular displacement of $0.06 \mathrm{rad}$ ). At this point the

Address for reprint requests: Dr GM Halmagyi, Eye and Ear Unit, Department of Neurology, Royal Prince Alfred Hospital, Camperdown, 1050, NSW, Australia.

Received 18 January 1983 and in revised form 16 March 1983. Accepted 16 April 1983. fall was decelerated by strong rubber straps which brought the apparatus to rest in two or three oscillations. The total downwards displacement of the couch when first released, including the breaking phase depended upon the weight of the individual, for example approximately $80 \mathrm{~cm}(0.4 \mathrm{rad})$ for a $60 \mathrm{~kg}, 170 \mathrm{~cm}$ female subject. As measured by recordings from the accelerometer the peak downwards acceleration was attained in $5 \mathrm{~ms}$ and lasted for $15 \mathrm{~ms}$. Patients and subjects all thought that the apparatus gave a smooth, comfortable ride.

\section{Subjects and patients}

Five young volunteers with clinically normal visual, auditory, and neurological function were the control subjects. Five patients without any evidence of horizontal or vertical semi-circular canal function comprised the "canaldefective" group. None had nystagmic responses to ice water caloric stimulation or to $200 \% \mathrm{~s}^{2}$ impulsive rotation; all five experienced head movement induced oscillopsia but had normal hearing. One of the five had lost canal function during treatment with intravenous gentamicin; the cause of the canal failure in the other four patients was unknown. Two other patients, both with endolymphatic hydrops due to congenital syphilis were also tested: neither had any evidence of canal function, one had no hearing at all and the other had some residual hearing.

\section{Protocol}

The subjects or patients lay comfortably in the couch with the head elevated about $20^{\circ}$ from the horizontal plane (fig 1). In this position the plane of the horizontal canals and the utricular maculae are oriented vertically and gravity is acting in shear on the utricle. One pair of $\mathrm{Ag}-\mathrm{AgCl}$ skin electrodes recorded the latency of the orbicularis oculi EMG (that is eyeblinks) from each side; a third pair supramaximally stimulated one supraorbital nerve. In some 


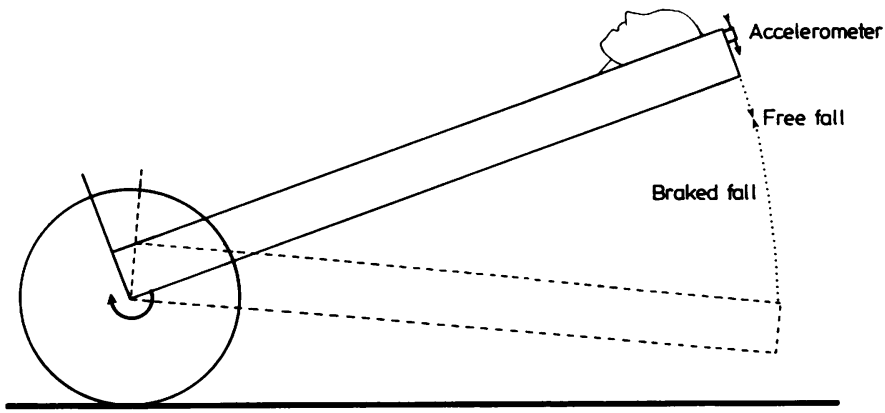

Fig 1 The experimental apparatus is shown in elevated position: continuous lines and lowest position during dropping: dotted lines

subjects and patients the surface EMG of other muscles such as sternomastoid and quadriceps was also recorded. (A linear accelerometer (Endevco Model 2265C with a bandwidth $>\mathrm{kHz}$ ) attached to the head of the bed recorded the fall). During the initial experiments subjects were blindfolded and listened to $75 \mathrm{~dB}$ white noise through headphones; later we found that blindfolding and soundmasking made no difference to the results, so the study was conducted in dim light with standard laboratory background noise.

The couch would be unpredictably released into free-fall by an assistant standing behind it. There was at least one minute between drops and each drop was preceded or followed by a shock to the supra-orbital nerve. The interval between the drop and shock was varied between 10 and $800 \mathrm{~ms}$. When the shock followed the drop, the timing was controlled using a variable delay stimulator triggered by the initial high frequency transient from the accelerometer. No automatic method of timing the interval between the stimuli when the shock preceded the drop was available and this had to be controlled in a hit and miss fashion by the operator who triggered the stimulator and jerked the cleat in sequence. In fact, with a little practice, one could become adept at producing various time delays and a fair sample of the range required could be attained within a few trials.

\section{Results}

\section{Supraorbital nerve shock}

Supraorbital nerve shock produced two wellrecognised EMG components: ${ }^{4}$ an ipsilateral early component starting $10-15 \mathrm{~ms}$ after the shock $\left(R_{1}\right)$ and a bilateral later component starting $35-45 \mathrm{~ms}$ $\left(R_{2}\right)$. The $R_{2}$ component causes the observable blink and is therefore called the eyeblink reflex. The latency to the onset of the $R_{2}$ component in normal subjects was $36.8 \pm 5.4 \mathrm{~ms}(113$ trials $)$ and $37.4 \pm$ $5.3 \mathrm{~ms}$ ( 37 trials) in the seven patients. There is no significant difference between the results in these two groups.

\section{Sudden free-fall}

In a total of 179 drops, each normal subject invariably showed orbicularis oculi EMG activity starting within $45 \mathrm{~ms}$ of the drop (39.3 $\pm 5.6 \mathrm{~ms})$ (fig 2). In some drops, but not in others, EMG activity was
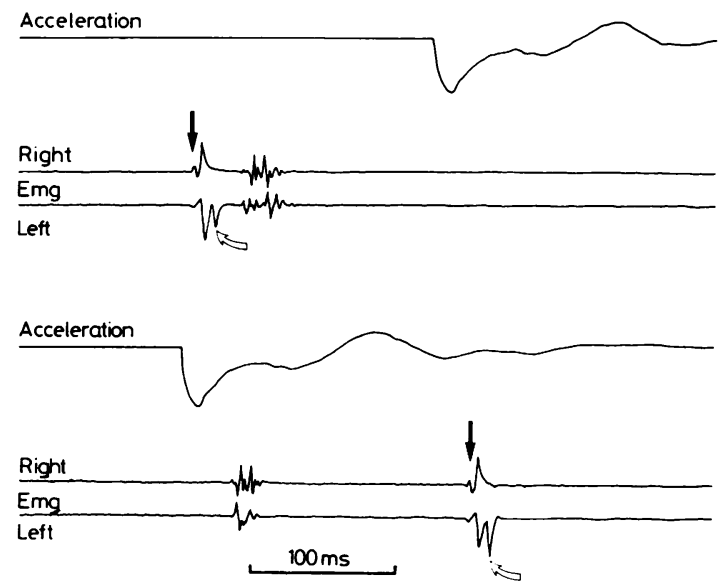

Fig 2 Orbicularis oculi EMG response is of a normal subject and to supraorbital nerve shock and to sudden drop. Acceleration equals output of linear accelerometer attached to head of couch. EMG is from surface of right and left orbicularis oculi. Top traces: a shock to the left supraorbital nerve (stimulus artefact indicated by filled arrow) is followed by an ipsilateral $R_{1}$ response (open arrow) and a bilateral $R_{2}$ response starting at about $40 \mathrm{~ms}$ after the stimulus. A sudden drop $180 \mathrm{~ms}$ after the supraorbital nerve shock fails to elicit any EMG response in either orbicularis oculi. Bottom traces: a sudden drop is followed about $40 \mathrm{~ms}$ later by the onset of EMG activity in both orbiculares oculi. A supraorbital nerve shock $200 \mathrm{~ms}$ after the drop (stimulus artefact indicated by closed arrow) is followed only by ipsilateral $R_{1}$ response (open arrow) and no $R_{2}$ response in either orbicularis oculi. 
also detected in quadriceps femoris and sternomastoid. In a total of 50 drops each canal-defective patient invariably showed orbicularis oculi EMG activity starting within $45 \mathrm{~ms}$ of being dropped (40.0 $\pm 4.1 \mathrm{~ms}$ ). There is no significant difference between the results in these two groups. Neither patient with congenital syphilis showed any orbicularis oculi EMG response within $100 \mathrm{~ms}$ of being dropped.

\section{Refractory period}

For $250-300 \mathrm{~ms}$ after a shock-induced eyeblink reflex neither normal subjects nor canal-defective patients showed a drop-induced eyeblink reflex: that is, there was never a drop-induced eyeblink reflex under $250 \mathrm{~ms}$ and invariably a normal, dropinduced eyeblink reflex over $300 \mathrm{~ms}$. Similarly, after a drop-induced eyeblink reflex, neither normal subjects nor canal-defective patients showed a shockinduced reflex for $250-300 \mathrm{~ms}$ (fig 2). In contrast, both patients with congenital syphilis showed normal latency shock-induced eyeblink reflexes within $250 \mathrm{~ms}$ of being dropped, at all latencies measured. The $R_{1}$ component of the shock-induced reflex was, as expected, unaffected by a preceding drop.

\section{Discussion}

In animals, bilateral labyrinthectomy or bilateral vestibular neurectomy both abolish early EMG responses to free-fall, whereas plugging of semicircular canals does not, indicating by exclusion that the EMG response originates in the otoliths. ${ }^{3}$ Our results also can best be explained by assuming that the orbicularis oculi EMG response to free-fall originates in the otolith. Normal responses in the five canal-defective patients, indicates that unless the vestibulo-ocular reflex and the responses to free-fall originate in a completely different set of receptors on the crista ampullaris, the response to free-fall cannot originate in the semi-circular canals. One of these five patients had lost canal function from amino-glycoside toxicity and it is known that the otolith receptors are less vulnerable to aminoglycosides than the semi-circular canal receptors. ${ }^{4}$ It is therefore not unexpected that otolith function was preserved in this patient. The cause of absent canal function was not known in the other four patients but the otolith epithelium may have been spared just as in the patients with aminoglycoside toxicity. Absent eyeblink reflexes to free-fall in the two patients with congenital syphilis suggests that their otoliths were, unlike the otoliths of the canal defective groups, not spared. The temporal bone pathology in congenital syphilis supports this suggestion: there is hydrops of the utricle and saccule, as well as atrophy of the organ or corti, cristae, cochlear and vestibular nerves. ${ }^{5}$ These explanations also seem valid for the four patients Greenwood and Hopkins studied. ${ }^{1,2}$ In two of their patients loss of canal function was due to aminoglycosides and in two the cause was unknown. Two had no canal responses and no EMG response to free-fall and two had normal canal and free-fall responses. Interestingly, we re-studied one of the latter group (WB) and found that he had lost all evidence of canal function but still had normal eyeblink responses to free-fall.

The results of the cross habituation experiments confirmed previous suggestions that the eyeblink reflex to free-fall is part of the startle reflex, elicited in this case by vestibular stimulation..$^{1,2}$ The eyeblink is the most durable component of the startle reflex: for example, it is present even in experienced pistol shooters each time they fire. ${ }^{6}$ The startle reflex has inhibitory as well as excitatory components: after one startle reflex has been elicited there is a refractory period during which a further stimulus will not elicit further response. ${ }^{7}$ Fox studied the excitatory and inhibitory components of the eyeblink reflex to loud tone-bursts and to painful electric shocks. ${ }^{7} \mathrm{He}$ found that with either stimulus, after initial EMG activation there is a much longer inhibitory period during which the eyeblink reflex to a subsequent stimulus of the same or different modality is completely blocked. In our experiments a shock-induced eyeblink reflex was followed by a $250-300 \mathrm{~ms}$ refractory period during which a drop could not induce an eyeblink reflex. These results, identical with Fox's tone-bursts and shocks, confirm previous suggestions that there is a common polysynaptic brainstem circuit for startle reflexes and that this circuit has access to auditory, somato-sensory, vestibular and visual inputs. ${ }^{8-11}$

Here we have devised a clinical technique utilising this brainstem circuit to test, albeit grossly, the integrity of otolith inputs. We have found relatively preserved otolith function in some patients who have no evidence of semi-circular canal function. The technique could now be used to test residual otolith function in patients who have lost canal function through labyrinthine injury.

\section{References}

' Greenwood R, Hopkins A. Muscle responses during sudden falls in man. $J$ Physiol 1976;254:507-18.

${ }^{2}$ Greenwood R, Hopkins A. Landing from an unexpected fall and a voluntary step. Brain 1976;99:375-86.

${ }^{3}$ Watt DGD. Responses of cats to sudden falls: an otolith-originating reflex assisting landing. $J$ Neurophysiology 1976;39:257-65.

${ }^{4}$ Lindeman HH. Regional differences of vestibular sensory epithelium to ototoxic antibiotics. Acta 
Otolaryngol 1969;67:177-89.

s Karmody CS, Schuknecht HF. Deafness in congenital syphilis. Archs Otolaryngol 1966;83:18-27.

${ }^{6}$ Landis C, Hunt WA. The Startle Pattern. New York: Farrar and Rhinehart, 1939.

${ }^{7}$ Fox JE. Excitatory and inhibitory components of the eye blink response to startle evoking stimuli, studied in the human subject. Electro-encephalography and Clinical Neurophysiology 1978;44:490-501.

${ }^{8}$ Fox JE, Wolstencroft JH. The reduced responsiveness of neurons in the nucleus reticularis gigantocellularis following their excitation by peripheral nerve stimula- tion. J Physiol (Lond) 1976;258:687-704.

${ }^{9}$ Groves PM, Wilson CJ, Boyle RD. Brainstem pathways, cortical modulation and habituation of the acoustic startle response. Behavioural Biology 1974;10:391418.

${ }^{10}$ Shanani BT, Young RR. Blink reflexes in orbicularis oculi, in: JE Desmedt (ed.) New Developments in Electromyography and Clinical Neurophysiology, vol 3. Karger, Basel, 1973.

"Szabo I, Hazafi K. Elicitibility of the acoustic startle reaction following brainstem lesions. Acta Physiol Acad Sci (Hung) 1965;27:155-65. 